\author{
by Jacques Vigué \\ Laboratoire Collisions, Agrégats, Réactivité UMR 5589, CNRS - Université de Toulouse, UPS \\ DOI: https://doi.org/10.1051/epn/2019501
}

\title{
Some comments on the historical paper by H. Schmidt-Böcking "The Stern-Gerlach experiment re-examined by an experimenter" (EPN 50/3 pp. 15-19)
}

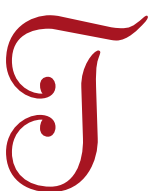

his paper is very interesting, with a large amount of poorly known or fully unknown details about this experiment. However, I would like to correct two imprecisions and add a small complementary information:

Bottom of page 15: in the sentence "Using the Molecular Beam Method (MBM) that he invented in 1919...", H. Schmidt-Bocking attributes to Stern the invention of the Molecular Beam Method. However, it is well known that the first molecular beam was built by L. Dunoyer [1] in 1911 who used it in the following years to observe the resonance fluorescence of sodium atom.

Middle of the second column of page 16: "As was typical of all experiments he [=Stern] performed, he carefully calculated the required conditions (the beam collimation parameters, the strength of the magnetic field, etc.) in order to be able to resolve, from the deflected beam, the tiny transverse momentum transfer due to the existence of an internal atomic magnetic moment (fig.1)". This sentence which refers to Stern 1921 paper[2] is somewhat misleading. The magnetic field gradient considered by Stern was $10^{4}$ Gauss per centimeter ( $10^{2} \mathrm{~T} / \mathrm{m}$ in SI units) and the calculated beam deflection was $1 / 100 \mathrm{~mm}$, a lot too small to be detected. This is due to the largely underestimated value of the magnetic field gradient and the experiment was a success because the gradient was at least 10 times larger. One may wonder why Stern has made such a large underestimation of the magnetic field gradient...

The complementary information is a note in Stern 1921 paper which states (text taken from the English translation) "Mr. W. Gerlach and I have been occupied for some time with the realization of this experiment. The reason for the present publication is the forthcoming paper by Messrs. Kallmann and Reiche concerning the deflection of electrical dipolar molecules in an inhomogeneous electric field. As I understand from the proofs, which were most kindly sent to me, our considerations are mutually complementary..... This note proves that the competition was strong and, at the same time, authors were kindly communicating the proofs of their papers to possible competitors! -

\section{References}

[1] L. Dunoyer, Comptes Rendus Acad. Sc. 152, 592 (1911).

[2] O. Stern, Z. Phys. 7, 249 (1921) which is available in an English translation in O. Stern, Z. Phys. D 10, 114 (1988) 


\section{ANSWER by Horst Schmidt-Böcking}

First I am happy and thankful that there are readers who read papers carefully and are willing to come in contact with the author.

To the history of the so-called molecular beam method MBM:

I presented in my paper the view of an experimental atomic physicist, who is performing with his group since several decades high resolution momentum imaging of atomic particles or fragments. Thus I see an important difference in the early history of the atomic beam experiments performed by Dunoyer and those performed 8 years later by Stern. Stern used the atomic beam of Dunoyer but he ennobled it to a precision method measuring tiny momentum transfers. This is for me really the beginning of the so-called MBS.

But Dunoyers work was clearly important for Stern and he mentioned it in his first publication on the Maxwell-Boltzmann velocity distribution measurement (O. Stern, Eine direkte Messung der thermischen Molekulargeschwindigkeit. Z. Physik 2, 49 (1920)). We will publish in October in the German "Journal für Physik) an article, which begins with the work of Dunoyer but then the article describes the basic new features of Otto Sterns MBM.
Stern was indeed in close contact with people in the Kaiser Wilhelm Institut für Physikalische Chemie in Berlin (today the Fritz Haber Institut) and knew what Reiche and Kallmann were planning to do (electric dipole moment). Therefore Stern published very early his idea how to perform a similar experiment deflecting atoms by the interaction of the inner atomic magnetic moment with an inhomogeneous magnetic field (the idea to use inhomogeneous fields was Madelungs idea, see interview of Stern in 1961 by Jost). In Sterns first publication (Otto Stern, Ein Weg zur experimentellen Prüfung der Richtungsquantelung im Magnetfeld. Z. Physik 7, 249 (1921)) his calculation was based on a magnetic field strength of about 10.000 Gauss $/ \mathrm{cm}$ thus the predicted deflection was only $0.01 \mathrm{~mm}$, but later in the real experiment of Feb. $7^{\text {th }}$ to $8^{\text {th }} 1922$ Gerlach reached fields of about 200.000 Gauss/cm, which yielded deflections of $0.1 \mathrm{~mm}$ or even a little more (see Walther Gerlach und Otto Stern, Über die Richtungsquantelung im Magnetfeld. Ann. Physik 74, 673 (1924) and Walther Gerlach, Über die Richtungsquantelung im Magnetfeld II, Annalen der Phys. 76, 163 (1925)).

\section{A Primer in Photoemission Concepts and Applications}

\section{De Antonio Tejeda et Daniel Malterre}

Photoemission is a spectroscopic technique to study the physicochemical properties of surfaces as well as their electronic properties, since it allows you to determine the band structure of the materials.

This book introduces the basic concepts of photoemission: core level and valence band photoemission, together with many recent developments on current topics. Two levels of reading are presented: an elementary primer based on a mono-electronic approach to qualitatively understand the interest of this spectroscopy and a deeper level supported on a many-body approach that allows you to get access to the interactions at the origin of the electronic properties of condensed matter.

This book addresses a broad span of readers, from undergraduate students for the more elementary aspects, to the research scientists specialized in the technique for the concepts and the application examples.

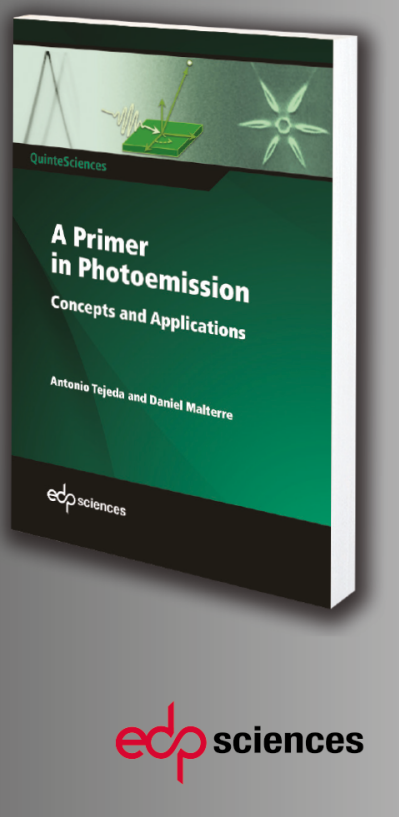

